\title{
PATRONES DE DISTRIBUCIÓN DE LAS MARIPOSAS DIURNAS EN PORTUGAL (LEPIDOPTERA, PAPILIONOIDEA, HESPERIIDAE, ZYGAENIDAE)
}

\author{
P. Garcia-Pereira1 ${ }^{\text {, E. García-Barros² y M. Munguira² }}$
}

\begin{abstract}
RESUMEN
La información corológica (datos de bibliografía, colecciones e inéditos) sobre las 143 especies de mariposas (Superfamilia Papilionoidea, y Familias Hesperiidae y Zygaenidae) de Portugal continental se ha reunido en una base de datos. Esta se emplea para definir patrones de distribución, aplicando el análisis factorial de correspondencias (AFC) a las matrices de frecuencias y de presencia-ausencia, basadas en una rejilla UTM de 50 x $50 \mathrm{~km}$. En primer lugar, se identifican los elementos y regiones faunísticas mediante la aplicación automática del análisis de clasificación a los resultados del AFC. En una segunda fase, se establece la relación entre los patrones obtenidos y un conjunto de variables ambientales, para determinar los posibles factores asociados a las regularidades detectadas. La temperatura media anual es el principal factor explicativo del gradiente latitudinal observado. Queda bien definida una región norte interior, de altitudes medias elevadas, y limitada hacia el sur por la Serra da Estrela. Esta área presenta mayor riqueza de especies que el resto del territorio, e incluye el Parque Natural de Montesinho, donde se localiza la máxima diversidad de mariposas del país. En la mitad meridional del país, destaca la región del Algarve, con un conjunto de especies de requisitos biológicos típicamente mediterráneos.
\end{abstract}

Palabras clave: Mariposas, Lepidoptera, Portugal, Biogeografía, Diversidad, Análisis de correspondencias.

\section{ABSTRACT \\ Patterns of distribution of butterflies and burnets in Portugal (Lepidoptera, Papilionoidea, Hesperiidae, Zygaenidae)}

An extensive faunistic database has been set up gathering bibliographic information, data from scientific collections and other relevant unpublished data from the 143 species of continental Portuguese butterflies and burnets (Superfamily Papilionoidea, Families Hesperiidae and Zygaenidae). This database was used to define distribution patterns through the application of factorial correspondence analysis (FCA) to the matrices of frequency and presence-absence data in a $50 \times 50 \mathrm{~km}$ UTM grid. The identification of faunistic elements and regions was obtained through an automatic application of the classification analysis to the FCA results. In a second instance, we used the same methodology to establish the relationship between previously obtained patterns and a set of environmental variables, in order to determine the factors related to the observed classes. The annual average temperature proved to be the best factor to explain the observed latitudinal gradient in the butterfly distribution. The results show a well-defined northern interior region of high average altitudes and limited in the South by the Serra da Estrela. This area has the largest species richness and hosts the Montesinho Natural Park, which is the most diverse in the country. In the southern half of Portugal, the Algarve deserves a special mention for its group of species with typical Mediterranean requirements.

Key-words: Butterflies, Burnets, Lepidoptera, Portugal, Biogeography, Diversity, Correspondence analysis.

Tagis- Centro de Conservação das Borboletas de Portugal. Museu Bocage. Rua da Escola Politécnica, 58. 1269-102 Lisboa - Portugal.

Departamento de Biología (Zoología). Universidad Autónoma de Madrid. E-28049 España. 


\section{Introducción}

Dennis et al. (1991, 1995a, 1995b, 1998) y Dennis \& Williams (1995) han estudiado la estructura de la fauna de mariposas de la región Paleártica occidental aplicando el análisis multifactorial a los datos de los mapas de Higgins \& Riley (1983). De acuerdo con estos autores, el espacio geográfico considerado se divide en dos grandes regiones delimitadas latitudinalmente por la cordillera de los Alpes. El norte de Europa constituye una vasta zona homogénea, caracterizada por especies de amplia distribución. En contraposición, la mitad meridional (que comprende las montañas del sur de Europa y la región Mediterránea), presenta una estructura compleja, con mayor número de unidades faunísticas y de elementos endémicos. La subdivisión de la región Mediterránea tiene estructura longitudinal. Su porción occidental, que incluye la Península Ibérica y el Norte de África, contiene unidades claramente diferenciadas del sector mediterráneo oriental.

Esta estructura refleja la secuencia de fluctuaciones ambientales que tuvieron lugar al final del Pleistoceno, comunes a otros taxones (p.ej.: de Jong, 1998; Hewitt, 1999). La influencia de estos acontecimientos históricos en la distribución de las mariposas ha debido operar a través de dos características esenciales de estos animales: gran sensibilidad a alteraciones climáticas, en tanto que ectotermos (y en especial la temperatura: Dennis et al., 1991; Dennis, 1993), y estrecha dependencia de la vegetación, en tanto que fitófagos estenófagos. $\mathrm{La}$ alteración del área de distribución constituye una respuesta rápida que permite la supervivencia de las especies en periodos de inestabilidad ambiental (Lobo, 1999). La eficacia de esta adaptación espacial está principalmente condicionada por la capacidad de dispersión de los organismos. En el caso de las mariposas, la distribución real de las plantas nutricias impone límites a la capacidad de dispersión. Por ello, muchos de estos insectos pueden ser considerados como de limitada capacidad dispersiva, a pesar de sus posibilidades teóricas debidas al vuelo (Thomas, 1989).

Según Dennis et al. (1991), los ciclos glaciales afectaron las especies diferencialmente, dependiendo de sus requisitos climáticos. Durante los máximos glaciales, la mayoría de las especies sufrió la extinción de sus poblaciones septentrionales, quedando su área de distribución restringida a los hábitats adecuados localizados en refugios a menores latitudes. Estas poblaciones meridionales quedaron aisladas en los periodos glaciales, debido al efecto de barrera que debieron ejercer las cadenas montañosas, dándo- se las condiciones para que se verifiquen fenómenos de divergencia genética y especiación. Los cortos periodos interglaciales, como el presente, se caracterizan por la expansión de las áreas de distribución de las especies con mayores tolerancias climáticas: recolonización de latitudes altas, y de mayores elevaciones altitudinales en latitudes bajas.

\section{LA Península IBÉRICA}

La Península presenta un total de 239 especies de mariposas (no considerando la familia Zygaenidae), siendo España el tercer país europeo por su riqueza especifica (tras Italia y Francia: Kudrna, 2002). De este modo, constituye uno de los centros de diversidad, al tiempo que reúne las condiciones para la continuación del proceso evolutivo (heterogeneidad de hábitats, estabilidad, alopatría), y para la recuperación a largo plazo de la fauna del norte de Europa (Dennis \& Williams, 1995). Actualmente están censadas 16 especies endémicas. Pero se reconoce un importante número de casos que implican diferenciación genética infraespecífica en relación con poblaciones más septentrionales (Munguira, 1995), o especies desdobladas recientemente (ejemplos en De Jong, 1972; Olivares et al., 1999; Mazel \& Leestmans, 1999). El territorio peninsular alberga, además, los límites de distribución y posibles áreas de contacto de pares de especies de origen presumiblemente reciente, como por ejemplo Carcharodus alceae (Esper, 1780) y C. tripolinus (Verity, 1925) (De Jong, 1974, 1978), Lycaena tityrus (Poda, 1761) y L. bleusei (Oberthür, 1884) (Vives Moreno, 1994; Munguira et al., 1997), Melanargia galathea (L., 1758) y M. lachesis (Hübner, 1790), Pseudo-philotes baton (Bergsträsser, 1779) y $P$. panoptes (Hübner, 1808).

Dadas las condiciones mencionadas, sería de evidente interés determinar regularidades en los patrones de distribución ibéricos de las mariposas. Esto no ha sido posible hasta la fecha, debido a la carencia de un atlas corológico detallado referido al territorio peninsular. Los trabajos existentes, basados en unidades de área operativas de tamaño grande (cuadrados con lados de $100 \mathrm{~km}$, o de un grado geográfico, p. ej.: Martín \& Gurrea, 1990; Gutiérrez, 1996; GarcíaBarros et al., 2002) indican dos patrones básicos más o menos patentes. En primer lugar, un gradiente de diversidad SO-NE, que supone un aumento de número de especies conforme disminuye la distancia a los Pirineos, y que ha sido interpretado como evidencia del "efecto península" (Martín \& Gurrea, 1990; Gutiérrez, 1996). Y en segundo lugar, un aumento de la diversidad en relación con el aumento de altitud promedio, que hace que las montañas representen los 
principales puntos calientes (hotspots) en la diversidad y tasa de endemicidad de estos lepidópteros en Iberia (Martín \& Gurrea, 1990; García-Barros et al. 2002). Estas regularidades son interesantes y en cierta medida contradictorias. El mencionado efecto peninsular implica un origen reciente, dispersivo, de la fauna ibérica de este grupo de insectos, contrapuesto a la existencia de refugios glaciales en el área mediterránea occidental, tal vez con excepción de los propios Pirineos, y no explica la relativa riqueza faunística de otras cadenas montañosas.

La coincidencia de varios gradientes macroambientales que operan en la Península de norte a sur o de este a oeste (geología, latitud, elevación e irregularidad, climatología, vegetación), dificulta por el momento la labor de encontrar y explicar una causa universal que explique los patrones de distribución de estos animales. Una posibilidad interesante sería estudiar una franja del territorio suficientemente grande en la que alguno de estos factores careciese de efecto relevante y este podría ser el caso de Portugal. La posición geográfica del territorio portugués, en el extremo sudoeste de la Península Ibérica, así como sus altitudes relativamente bajas, excluyen la presencia de elementos endémicos ligadas a áreas de montaña (de hecho, de acuerdo Dennis et al., 1991, la fauna portuguesa está constituida mayoritariamente por especies de amplia distribución en Europa). Como resultado del trabajo realizado por la primera firmante para la preparación de su Tesis Doctoral, se ha reunido una importante cantidad de información georeferenciada sobre la corología de las mariposas diurnas de Portugal. Este material permite abordar alguno de los extremos antes mencionados, y como mínimo, realizar una primera tipificación (basada en métodos estadísticos objetivos) de esta parte de la Península de acuerdo con su fauna lepidopterológica.

En consecuencia, este estudio pretende abordar dos objetivos: En primer lugar, describir los patrones biogeográficos subyacentes a la distribución de las especies en el territorio continental de Portugal, y en especial definir las unidades y regiones faunísticas del país sustentados por la compilación de la información faunística realizada. En segundo lugar, identificar los principales factores macroambientales relacionados con los patrones encontrados.

\section{Metodología}

\section{DATOS Y ESCALA DE REPRESENTACIÓN}

La información faunística utilizada en este estudio resulta de la compilación exhaustiva de datos de

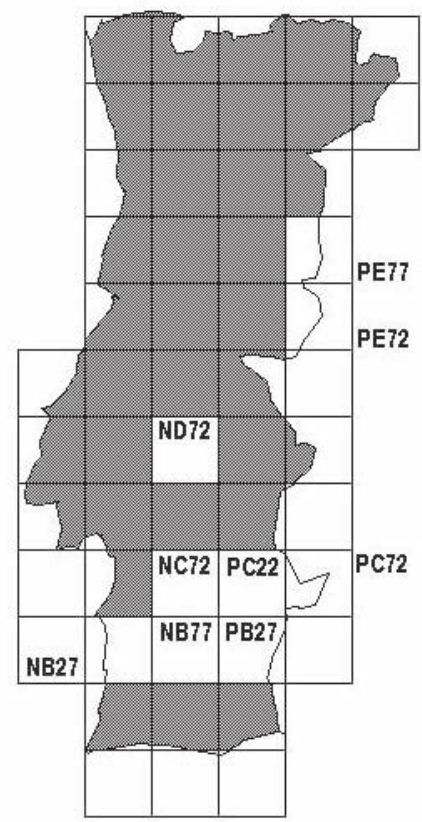

Fig. 1.- Universo geográfico utilizado en este estudio. En gris se muestran las 36 cuadrículas UTM de 50 x $50 \mathrm{~km}$ $\left(\mathrm{UTM}_{50}\right)$ analizadas. En blanco se señalan las cuadrículas $\mathrm{UTM}_{50}{ }_{50}$ excluidas por presentar un nivel de cobertura faunística inferior al $25 \%$.

Fig. 1.- Geographic universe used in this study. The 36 analyzed squares of the $50 \times 50 \mathrm{~km}$ UTM grid are shown in grey. The white squares represent the $\mathrm{UTM}_{50}$ excluded for presenting a level of faunistic coverage below $25 \%$.

colecciones, bibliografía y comunicaciones personales, reunidos en una base de datos faunística de las mariposas diurnas de Portugal (Garcia-Pereira, inédito; Maravalhas et al., 2003). Esta comprende 24649 registros correspondientes a 143 especies. Los taxones implicados son la superfamilia Papilionoidea, la superfamilia Hesperioidea (Familia Hesperiidae), y la Familia Zygaenidae, y los registros se refieren a cuadrículas UTM de $10 \times 10 \mathrm{~km}$ $\left(\mathrm{UTM}_{10}\right)$. Todo el territorio portugués se enmarca en las zonas $29 \mathrm{~T}$ y $29 \mathrm{~S}$. El conjunto de estos datos corológicos cubre aproximadamente mitad del territorio (466 $\mathrm{UTM}_{10}$ de las 977 cuadrículas posibles). De acuerdo con el actual nivel de cobertura, se consideró como más adecuada la malla de 50 x $50 \mathrm{~km}$ $\left(\mathrm{UTM}_{50}\right)$ para detectar patrones generales (GarciaPereira, inéd.; García-Barros \& Munguira, 1999; 
García-Barros et al., 2000). De las 45 cuadrículas $\left(\mathrm{UTM}_{50}\right)$ que interesan el territorio, se descartaron aquellas en la que la cobertura faunística (proporción de $\mathrm{UTM}_{10}$ con datos faunísticos sobre el total de cada $\mathrm{UTM}_{50}$ ) resultó inferior al 25\%. Así, el universo geográfico considerado quedó constituido por 36 cuadrículas de $50 \mathrm{~km}$ de lado (Fig. 1).

Los datos de partida fueron organizados en dos matrices distintas: 1) Matriz de presencia/ausencia (valores de 0 y 1 para cada especie), y 2) matriz de frecuencias, en la que se computó para cada unidad geográfica el número de registros de cada especie, siendo un registro igual a uno o más ejemplares referidos en una misma localidad y fecha por un mismo colector. En el Apéndice 1 se presentan los códigos de las especies, que han sido utilizados para la presentación de los resultados (Fig. 2 y Fig. 5).

\section{VARIABLES AMBIENTALES}

La información relativa a la caracterización ambiental del territorio fue importada de la base de datos "Atlas do Ambiente" (DGA, 2000). Se seleccionó el siguiente grupo de variables climáticas, topográficas y geológicas: 1- temperatura media anual, 2- precipitación media anual, 3- número medio de días de precipitación, 4- altitud, 5- tipo de substrato geológico, y 6- $\mathrm{pH}$ del suelo. Se tomaron los valores de estas variables para las cuadrículas de $10 \times 10 \mathrm{~km}\left(\mathrm{UTM}_{10}\right)$, a los que se refiere la información faunística original, utilizando los sistemas de información geográfica Arc/Info y Arcview GIS (ESRI, 1998a; ESRI, 1998b). El vértice suroeste de cada cuadrícula se usó como referencia para la captación de estos datos, excepto en el caso de cuadrículas periféricas o de borde (costas, fronteras), caso en el se utilizó el vértice de la cuadrícula que quedase inscrito en el territorio portugués. La matriz de datos referida a la malla de $50 \times 50 \mathrm{~km}\left(\mathrm{UTM}_{50}\right)$ se construyó como un recuento de frecuencias de las variables relativas a sus unidades $\mathrm{UTM}_{10}$.

Teniendo en cuenta que las variables estaban definidas como categorías en la fuente original, fue necesario cierto esfuerzo de recodificación (ver Tabla 1). En general fue posible redefinir las categorías sin pérdida importante de representatividad y significado ecológico (de acuerdo con Ribeiro, 1986). Las dos primeras clases de temperatura media anual (Tm1 y Tm2) corresponden a las estaciones de montaña, únicos lugares en los que la temperatura media anual es inferior a $15^{\circ} \mathrm{C}$. En cuanto a la precipitación media, las zonas que reciben más de $1000 \mathrm{~mm}(\operatorname{Pr} 3$ y Pr4) pueden considerarse húmedas. Los niveles de 400 y $900 \mathrm{~m}$ constituyen los dos principales límites mediterráneos de altitud. La pri-
Tabla 1.- Valores y códigos de las clases en las seis variables ambientales utilizadas. Se añaden asimismo el número de cuadrículas UTM de 10 x $10 \mathrm{~km}$ de lado con información faunística para cada una de las clases ( ${ }^{\circ}$ UTM10).

Table 1.- Values of the classes of the six environmental variables used and the codes used for each of them. The number of $10 \times 10$ UTM squares with faunistic data is also added for each class ( $\left.\mathrm{N}^{\circ} \mathrm{UTM} 10\right)$.

\begin{tabular}{|c|c|c|c|}
\hline \multicolumn{3}{|c|}{ Variables ambientales } & \multirow{2}{*}{ No UTM10 } \\
\hline & Clases & Cód. & \\
\hline Temperatura media anual & $<12,5$ & Tm1 & 78 \\
\hline \multirow[t]{3}{*}{$\left({ }^{\circ} \mathrm{C}\right)$} & $12,5-15$ & Tm2 & 136 \\
\hline & $15-16$ & Tm3 & 122 \\
\hline & $>16$ & Tm4 & 130 \\
\hline Precipitación media anual & $<600$ & Pre1 & 102 \\
\hline \multirow[t]{3}{*}{$(\mathrm{mm})$} & $600-1000$ & Pre2 & 202 \\
\hline & $1000-4000$ & Pre3 & 86 \\
\hline & $>1400$ & Pre4 & 76 \\
\hline Días de precipitación anual & $<75$ & Dp1 & 159 \\
\hline \multirow[t]{2}{*}{ (valor medio) } & $75-100$ & Dp2 & 209 \\
\hline & $>100$ & Dp3 & 98 \\
\hline Altitud media & $1-400$ & Alt1 & 310 \\
\hline \multirow[t]{2}{*}{ (m) } & $400-900$ & Alt2 & 130 \\
\hline & $900-1700$ & Alt3 & 26 \\
\hline \multirow[t]{5}{*}{ Tipo de substrato } & Arenas (rio) & Arer & 68 \\
\hline & Arenas (mar) & Arem & 12 \\
\hline & Calizas & Cali & 88 \\
\hline & Pizarras & Piza & 160 \\
\hline & Granitos & Gran & 138 \\
\hline \multirow[t]{3}{*}{ pH de suelo } & Ácido & pHac & 402 \\
\hline & Alcalino & pHal & 36 \\
\hline & Neutro & pHne & 28 \\
\hline
\end{tabular}

mera cifra marca la desaparición de las plantas más termófilas (Alt2), y por encima de $900 \mathrm{~m}$ se encuentra un ambiente típico de montaña, con ausencia de especies vegetales mediterráneas espontáneas o cultivadas (Alt3). Para el tipo de substrato, se transformaron las 27 categorías disponibles en DGA (2000) en 5 grandes grupos, correspondientes a los principales tipos de rocas. Los datos presentados en la Tabla 2 permiten predecir que el $\mathrm{pH}$ del suelo tendrá escaso poder discriminante, ya que la gran mayoría del territorio presenta un substrato ácido.

\section{ANÁLISIS ESTADÍSTICOS}

La mayor parte de los datos disponibles representan variables de tipo discreto, por lo que se aplicó el análisis factorial de correspondencias (AFC) 
Tabla 2.- Composición específica de las tres regiones faunísticas propuestas.

Table 2.- Species composition of the three faunistic regions proposed.

\begin{tabular}{|c|c|c|c|}
\hline \multirow[b]{2}{*}{ Familia } & \multicolumn{3}{|c|}{ Regiones faunisticas } \\
\hline & Montesinho & rte interior & $\begin{array}{c}\text { Sur } \\
\text { Algarve } \\
\end{array}$ \\
\hline Zygaenidae & \begin{tabular}{|l} 
Adscita bolivari \\
Adscita schmidti
\end{tabular} & Adscita notata & $\begin{array}{l}\text { Zygaena hilaris } \\
\text { Zygaena occitanica } \\
\text { Zygaena rhadamanthus } \\
\text { Zygaena fausta } \\
\text { Zygaena lavandulae } \\
\end{array}$ \\
\hline Hesperiidae & Pyrgus alveus & $\begin{array}{l}\text { Hesperia comma } \\
\text { Ochlodes venata } \\
\text { Erynnis tages } \\
\text { Pyrgus armoricanus }\end{array}$ & $\begin{array}{l}\text { Gegenes nostrodamus } \\
\text { Syrichtus proto }\end{array}$ \\
\hline Pieridae & & & $\begin{array}{l}\text { Colias alfacariensis } \\
\text { Euchloe tagis }\end{array}$ \\
\hline Nymphalidae & $\begin{array}{l}\text { Brenthis ino } \\
\text { Brenthis daphne } \\
\text { Boloria dia } \\
\text { Erebia triaria } \\
\text { Satyrus actaea }\end{array}$ & $\begin{array}{l}\text { Nymphalis antiopa } \\
\text { Inachis io } \\
\text { Aglais urticae } \\
\text { Polygonia c-album } \\
\text { Argynnis paphia } \\
\text { Mesoacidalia aglaja } \\
\text { Fabriciana niobe } \\
\text { Fabriciana adippe } \\
\text { Boloria selene } \\
\text { Boloria euphrosyne } \\
\text { Melitaea cinxia } \\
\text { Melitaea athalia } \\
\text { Melitaea trivia } \\
\text { Melitaea parthenoides } \\
\text { Apatura ilia } \\
\text { Azuritis rectucta } \\
\text { Coenonympha arcania } \\
\text { Coenonympha glycerion } \\
\text { Hypophenele Iycaon } \\
\text { Melanargia russiae } \\
\text { Hipparchia alcyone } \\
\text { Brentesia circe } \\
\text { Arethusana arethusa } \\
\text { Libythea celtis }\end{array}$ & $\begin{array}{l}\text { Euphydryas desfontainii } \\
\text { Melitaea aetherie }\end{array}$ \\
\hline Lycaenidae & $\begin{array}{l}\text { Lycaena virgaureae } \\
\text { Hamearis lucina }\end{array}$ & $\begin{array}{l}\text { Lycaena tityrus } \\
\text { Lycaena alciphron } \\
\text { Glaucopsiche alexis } \\
\text { Maculinea alcon }\end{array}$ & \begin{tabular}{|l|} 
Cacyreus marshalli \\
Cupido lorquinii \\
Pseudophilotes panoptes \\
Pseudophilotes abencerragus
\end{tabular} \\
\hline
\end{tabular}

(Hill, 1974; Mercier et al., 1992). La clasificación subsiguiente fue efectuada automáticamente sobre los resultados del AFC, utilizando las coordenadas de las clases en los ejes extraídos. Este procedimiento permite crear tipologías tras verificar la estructura asociada a los datos iniciales, eliminando así la variabilidad inicial que no contribuye a definir patrones. Además, elimina la subjetividad inhe- rente a la selección de una medida de asociación por parte del investigador. Se utilizó la distancia euclídea para la construcción de los dendrogramas, dado que los ejes son ortogonales. Se aplicó el programa ANDAD versión 6.0 b (CVRM/IST, 2000) para la ejecución del AFC, mientras que la clasificación se realizó con el programa STATISTICA versión 5.1 (Statsoft. Inc., 1996). 
a
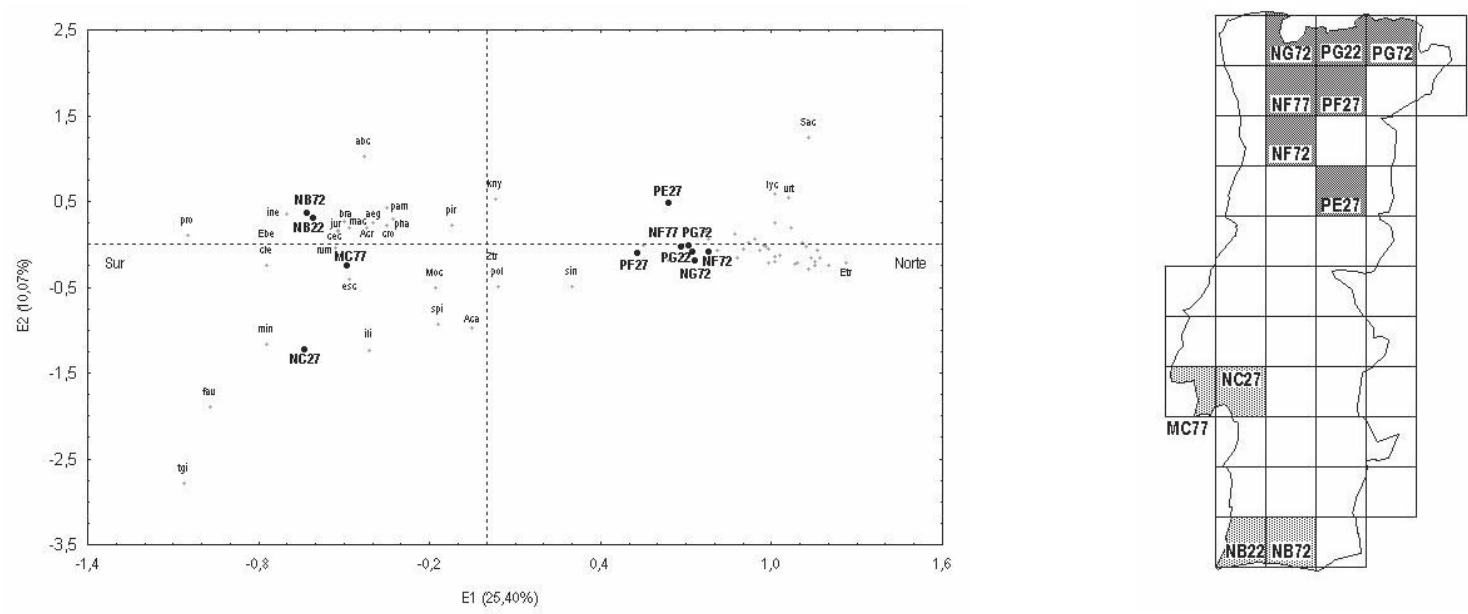

b
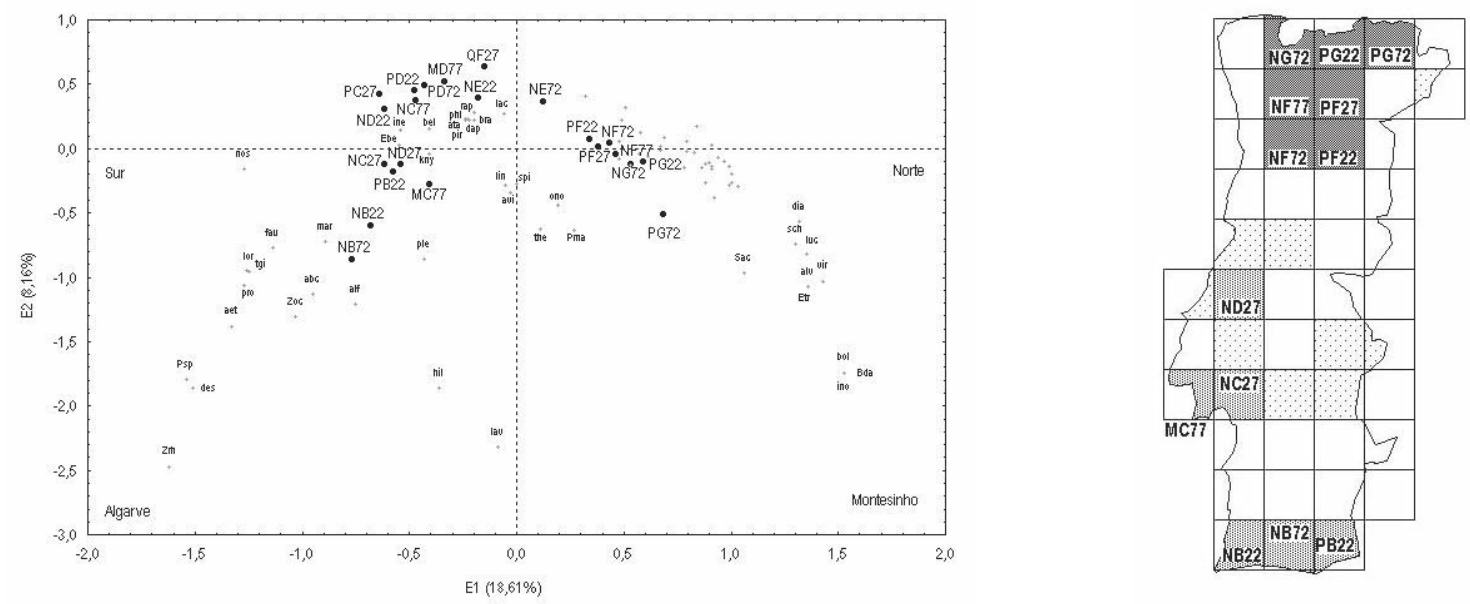

Fig. 2.- Proyección de las cuadrículas (puntos) y especies significativas (cuadrados) para la construcción de los dos primeros ejes extraídos del análisis factorial de correspondencias, a) datos de frecuencias, b) datos de presencia y ausencia. En los dos gráficos se omiten las abreviaturas de las especies características de la región norte. El color gris oscuro de los mapas indica las cuadrículas significativas del norte y el gris claro las del sur. En el mapa b, las cuadrículas significativas para la mitad positiva del eje $\mathrm{E}_{2}$ no incluidas en las dos categorías anteriores, están señaladas con una trama de puntos.

Fig. 2.- Projection of the significant squares (dots) and species (squares) for the construction of the two first axes obtained by the application of the factorial correspondence analysis, a) data on frequencies, b) presence-absence data. In both figures, the abbreviations of characteristic northern species are omitted. The dark gray in the maps represents the significant northern squares and the light gray the southern. In the map $\mathrm{b}$, the significant squares for the positive half of $\mathrm{E}_{2}$ axis not included in the two previous categories are shown with stapled pattern.

\section{Resultados}

\section{DATOS DE FRECUENCIAS}

Los dos primeros ejes extraídos del AFC, que explican de $35,5 \%$ del total de varianza original, son suficientes para traducir la estructura principal asociada a los datos originales. A partir de la interpretación de la posición relativa del conjunto de
$\mathrm{UTM}_{50}$ significativas en el plano definido por esos ejes (Fig. 2a), se verifica que el eje principal representa una clara división latitudinal, diferenciando cuadrículas pertenecientes al norte y sur del territorio. En la mitad norteña destaca un conjunto de cuadrículas muy próximas entre sí. Mientras, en el sur se definen el área cercana a Lisboa, y la región occidental del Algarve. El segundo eje produce una 


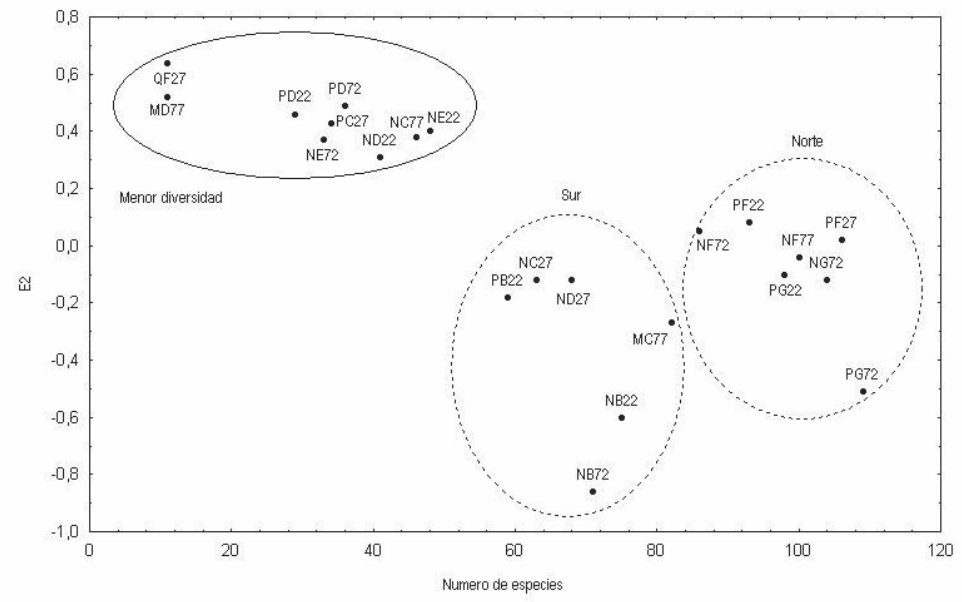

Fig. 3.- Relación entre las coordenadas del el segundo eje $\left(\mathrm{E}_{2}\right)$ tomado de la matriz de presencia-ausencia y el numero de especies conocido del conjunto de $\mathrm{UTM}_{50}$ significativos. El grupo de cuadrículas de la mitad positiva del eje presenta menor riqueza específica, correspondiendo a zonas del territorio con ausencias de especies. El numero de especies citadas permite separar las cuadrículas localizadas en la región norte y sur de Portugal.

Fig. 3.- Relationship between the significant $\mathrm{UTM}_{50}$ for the second axis $\left(\mathrm{E}_{2}\right)$ obtained with the presence-absence data and the total number of species. The group in the positive half of $\mathrm{E}_{2}$ has lower species richness, corresponding to areas where the absences of species are concentrated. The number of species allows the separation between squares in the north and south of the country.

separación dentro de la mitad meridional, aislando claramente la fauna característica de la Serra da Arrábida (NC27). Con relación al grupo del norte, la cuadrícula correspondiente a la Serra da Estrela (PE27) es la única que presenta una contribución significativa a este eje; su posición en la mitad positiva sugiere proximidad faunística al Algarve, lo que podría interpretarse como una un papel fronterizo de esa zona, entre las dos regiones definidas por el primer eje.

Al representar en el mismo plano la posición de las especies se observa un numeroso grupo concentrado alrededor de los $\mathrm{UTM}_{50}$ del norte. Etr $=$ Erebia triaria (De Prunner, 1798) y pro $=$ Syrichtus proto (Ochs., 1808) marcan los extremos septentrional y meridional en el gradiente latitudinal. Surgen también fuertes asociaciones con las sierras: en el sur, entre tgi= Euchloe tagis (Hübner, 1909), fau= Zygaena fausta (L., 1767), $\mathrm{min}=$ Cupido minimus (Fuessly, 1775) e ili= Satyrium ilicis (Esper, 1779), especialmente con la Arrábida; en el norte, entre $\mathrm{Sac}=$ Satyrus actaea (Esper, 1780), lyc $=$ Hypophenele lycaon (Kühn, 1774) y urt= Aglais urticae (L., 1758), y la Serra da Estrela. A pesar de que estas especies presentan una distribución más amplia, es en esas cuadrículas donde son más comunes. Por ejemplo, para Euchloe tagis cerca de $80 \%$ de los registros corresponden a la Serra da Arrábida, y el 92,5\% de los registros de $S$. actaea proceden de observaciones efectuadas en la Serra da Estrela (Garcia-Pereira, inédito).

En la zona central del gráfico se ubican las especies de amplia distribución en Portugal, próximas a los dos grupos de cuadrículas. De entre las especies extendidas por la mitad sur (sector positivo del eje) destacan $\mathrm{abc}=$ Pseudophilotes abencerragus (Hübner, 1808) y kny= Zizeeria knysna (Trimen, 1862). Las especies $\sin =$ Leptidea sinapis (L., 1758), $\mathrm{Ztr}=$ Zygaena trifolii (Esper, 1783), pol= Nymphalis polychloros (L., 1758) en la mitad norte, así como $\mathrm{Moc}=$ Melanargia occitanica (Esper, 1793), spi= Satyrium spini (Schiff., 1775), o Aca $=$ Anthocharis cardamines (L., 1758) en la mitad sur, se asocian a la cuadrícula NC27. Los restantes elementos colocados cerca de la mitad sur corresponden a especies distribuidas por todo el país, por ejemplo phl= Lycaena 


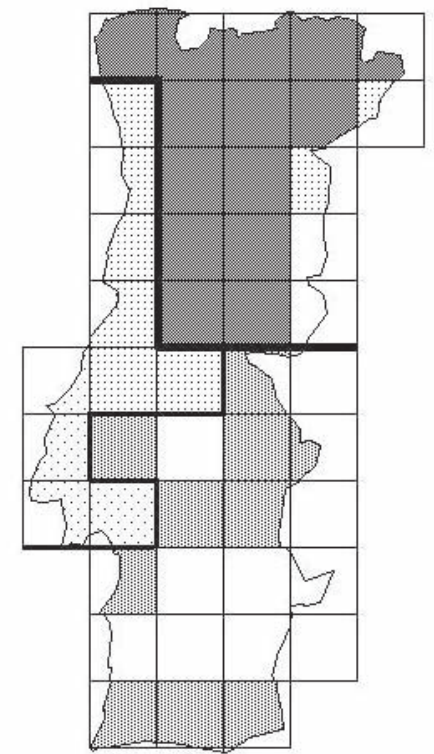

Fig. 4.- Regiones faunísticas obtenidas a partir del dendrograma construido con los resultados del análisis factorial de correspondencias (matriz de frecuencias; excluyendo Euchloe tagis y Satyrus actaea, ver texto).

Fig. 4.- Faunistic regions obtained through the application of a cluster analysis to the results of the factorial correspondence analysis (frequency data matrix, excluding Euchloe tagis and Satyrus actaea; see text).

phlaeas $(\mathrm{L} ., 1758)$ o jur= Maniola jurtina $(\mathrm{L} ., 1758)$. Esto refleja ciertas asimetrías de la información original. Por ejemplo, el volumen de datos del sur de Portugal (en particular el Algarve) es muy elevado (Garcia-Pereira, 1999); como consecuencia, algunas especies comunes y ampliamente distribuidas aparecen ligadas a esta zona.

\section{DATOS DE PRESENCIA-AUSENCIA}

Los resultados del análisis de la matriz de presencia-ausencia son, en términos generales, semejantes a lo anteriormente descrito (Fig. 2b). Sin embargo, se atenúan las asimetrías de cobertura antes mencionadas, al disminuir la dependencia de los datos del esfuerzo de muestreo. Los dos ejes principales explican el $26,77 \%$ de la varianza. El primero representa, como en el caso de la matriz de frecuencias, una segregación conforme a un gra- diente norte-sur. En relación con el segundo eje, tienen un peso significativo para la mitad negativa los $\mathrm{UTM}_{50}$ correspondientes a los extremos del territorio: Algarve occidental (NB22 y NB72) y Montesinho (PG72). A la parte positiva se asocia una serie de cuadrículas más dispersas (Alentejo o extremo nordeste). Su significado tiene que ver con la riqueza especifica. En la Fig. 3 se representa la relación entre las dos variables, y se observan tres grupos distintos. Las cuadrículas con coordenadas positivas en el Eje 2 presentan bajos valores de diversidad especifica (menos de 50 especies), correspondiendo a las zonas del territorio donde se verifican "ausencias" de las especies características de las restantes cuadrículas significativas. El número de especies por cuadrícula permite aún separar dos conjuntos en la mitad negativa del eje, correspondientes a las regiones sur y norte antes definidas. Se confirma que los $\mathrm{UTM}_{50}$ norteños constituyen áreas de mayor riqueza específica. La posición de las especies significativas en el plano evidencia igualmente este aspecto, observándose una mayor concentración de elementos en la mitad norteña (ver Fig. 2b). En el sur, con excepción de la fauna característica del Algarve, solamente surge aislada Gegenes nostrodamus (Fabricius, 1793) (nos), de distribución meridional en Portugal (Garcia-Pereira, inédito). Las especies hil= Zygaena hilaris (Och., 1808) y lav= Zygaena lavandulae (Esper, 1783) quedan aisladas en el centro del gráfico, dado que su presencia se ha señalado sólo de los dos extremos norte y sur de Portugal, el Algarve y Montesinho.

\section{UNIDADES Y REGIONES FAUNÍSTICAS}

Las regiones faunísticas formadas a partir de la interpretación de los resultados se muestra en la Fig. 4. Para la elaboración del dendrograma, se efectuó un nuevo ensayo de la AFC excluyendo Euchloe tagis y Satyrus actaea, que producen una distorsión en los resultados por la concentración de sus registros en únicamente dos cuadrículas de 10 $\mathrm{km}$ de lado. En el territorio portugués destaca esencialmente la fauna particular de la mitad norte, especialmente su zona interior desde el extremo del territorio hasta el limite meridional de la Serra da Estrela. En el resto del territorio la composición faunística es más homogénea, pero incluso así es aún posible individualizar una zona de transición situada en el litoral atlántico.

De acuerdo con el patrón descrito, podemos clasificar la fauna de mariposas portuguesa en tres grupos principales: Montesinho, Norte Interior y Algarve (Fig. 5). En la Tabla 2 se presenta la constitución específica de las unidades faunísticas, suge- 


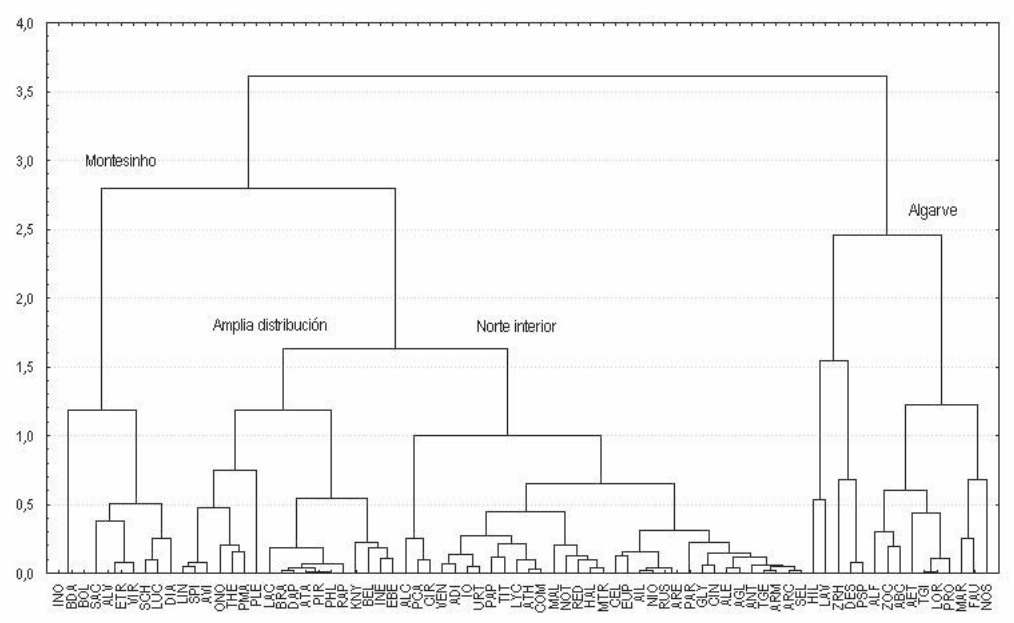

Fig. 5.- Unidades faunísticas obtenidas por el dendrograma elaborado a partir de los resultados del análisis factorial de correspondencias con la matriz de presencia-ausencia.

Fig. 5.- Faunistic groups obtained after the application of a cluster analysis to the results of the factorial correspondence analysis using the presence-absence data matrix.

rida por el dendrograma. La individualización de la región norte es debida esencialmente a la distribución restringida de un numeroso grupo de especies de Nymphalidae (especialmente Nymphalinae). Por otro lado, diversas especies de ziguenas parecen características de la región del Algarve.

\section{RELACIÓN CON LAS VARIABLES AMBIENTALES}

En la Fig. 6 se presenta la proyección de las clases de las variables que están relacionadas con el sistema de ejes basado en los datos de presencia-ausencia de las mariposas (contribuciones relativas para el primer eje superiores a 40\%). Temperatura y altitud son los principales factores explicativos de la segregación latitudinal. En especial, la temperatura parece ser un requisito fundamental para los elementos faunísticos del Algarve (Tm4). Así, se puede afirmar que el área de distribución de las especies características del sur, está condicionada de forma importante por la dominancia de un clima de tipo mediterráneo (temperaturas medias superiores a $12,5^{\circ} \mathrm{C}$ y menos de 75 días de precipitación). Los sustratos calizos parecen constituir un requisito adicional importante para este conjunto de especies. Por otro lado, las zonas de montaña, con temperaturas anuales medias más bajas (inferiores a $12,5^{\circ} \mathrm{C}$ ), constituyen las regiones preferidas por las especies características del norte.

\section{Discusión}

En Portugal Continental se observa una acentuada división latitudinal en la distribución de las especies de mariposas. Esta sectorización está particularmente relacionada con la variación de los valores medios de altitud y de temperatura anual. A partir de la información actualmente disponible, destaca especialmente la región faunística localizada en el norte interior, que se extiende desde el extremo del territorio hasta el limite meridional de la Serra da Estrela. Se observa igualmente una fuerte coincidencia geográfica entre los límites de esa región y las áreas de mayor altitud del país (Ribeiro, 1986). Allí se localizan, igualmente, las mayores densidades de especies, con un máximo en el área del Parque Natural de Montesinho. El resto del país presenta una fauna relativamente homogénea, caracterizada por una fauna comparativamente pobre.

Tanto los patrones de distribución como el número de especies parecen depender en gran medida de un gradiente de latitud y altitud, de forma coherente con lo observado en el resto del territorio peninsular (Martín \& Gurrea, 1990; Gutiérrez, 1996). Estos gradientes reflejan sobre todo la capacidad diferencial del medio ambiente para sustentar las especies, pero no es posible establecer una rela- 


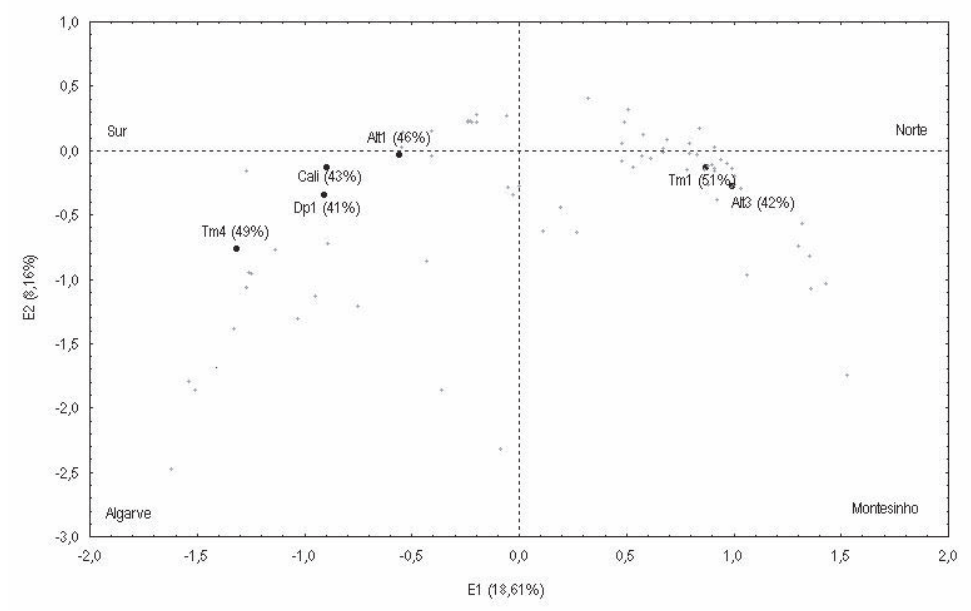

Fig. 6.- Proyección de las principales clases de las variables ambientales (contribuciones relativas para el primer eje superiores al 40\%) relacionadas con la distribución de las mariposas obtenida a partir de la matriz de presencia-ausencia. Los cuadrados corresponden a la posición de las especies de mariposas de la figura $2 \mathrm{~b}$.

Fig. 6.- Supplementary projection of the significant environmental classes (relative contribution for the first axis higher than $40 \%$ ) related to the distribution of butterflies obtained with the presence-absence matrix. The squares represent the position of butterfly species from the figure $2 b$.

ción causal directa entre ellos y el número de especies (Austin et al., 1996; Lobo et al., 2001). Por tanto, los factores climáticos (altamente correlacionados con altitud y latitud) se cuentan probablemente entre los principales condicionantes de la distribución de las mariposas en Portugal. La mitad septentrional se caracteriza por la presencia de un numeroso conjunto de elementos faunísticos de amplia distribución en Europa, y sin embargo de distribución restringida en Portugal. Por otro lado, en el Algarve se dan algunos elementos con requisitos biológicos típicamente mediterráneos.

Es cierto que las áreas de montaña han representado tradicionalmente un objetivo predilecto en la faunística entomológica (García-Barros \& Munguira, 1999; Martín \& Gurrea, 1999), y ello puede crear un artefacto en los datos debido a la mayor intensidad de recolección. Sin embargo, pensamos que este efecto ha de ser menor, ya que su principal causa es precisamente la mayor expectativa de diversidad faunística en esos puntos. Por lo demás, esta mayor variedad de especies de las áreas montañosas se relaciona con diversos factores. Uno es el solapamiento de las áreas de distribución de especies con diferentes tolerancias cli- máticas, y se explica por la gran heterogeneidad ambiental que las variaciones de altitud proporcionan (Gutiérrez \& Menéndez, 1995; Gutiérrez, 1997), y se observa igualmente en otros grupos de organismos (Myklestad \& Birks, 1993; Moreno Sainz et al., 1996; Castro Praga et al., 1996; Lumaret \& Lobo, 1997; García-Barros et al., 2002). En segundo lugar, la influencia antropogénica se hace sentir con más intensidad en zonas bajas y de relieve uniforme, por lo que las sierras mantienen mayor proporción de paisajes naturales (Lobo et al., 2001). En el territorio portugués en particular, se verifica además un desequilibrio entre el litoral y el interior, por que la costa presenta un mayor grado de densidad de población humana y degradación de la vegetación (Ribeiro, 1986).

Por último, es necesario hacer referencia a la influencia de los factores históricos. Algunos autores refieren la importancia de las cordilleras montañosas ibéricas en la presente distribución de las especies de mariposas centroeuropeas durante el Pleistoceno: como barreras geográficas y refugio, en los periodos glaciales, y como corredores de dispersión en los interglaciales (Martín \& Gurrea, 1990; Gutiérrez, 1996, 1997). Martín \& Gurrea 
(1990) sugieren que la actual distribución de las especies podría no representar una situación de equilibrio, sino parte de un período de expansión hacia el sur y suroeste de las áreas de distribución. Gutiérrez (1997) considera que, en los Picos de Europa, la colonización diferencial de los refugios existentes condiciona la presente composición y riqueza específica de las comunidades de las mariposas. Sin embargo, ambos estudios fueron realizados sin aislar completamente los factores históricos de los ecológicos, por lo que existe una dificultad metodológica en la base de sus consideraciones. Del mismo modo, en este trabajo, los métodos utilizados no permiten evaluar la importancia de los acontecimientos históricos en los patrones actualmente observados. No obstante, al extremo nordeste del territorio están asociadas una serie de especies con una distribución exclusiva, que no puede ser explicada por diferencias de clima o de altitud con relación al resto del norte interior. Corresponden a elementos característicos de las sierras Galaico-Cantábricas, como por ejemplo: Brenthis ino (Rottemburg, 1775), Brenthis hecate (Schiff., 1775) o Hamearis lucina (L., 1758). Es posible que la existencia de esta unidad faunística sea resultado de procesos de colonización diferencial de los refugios de las montañas portuguesas, que han conducido a cierta diferenciación con relación a las formaciones del Sistema Central Ibérico, como la Serra da Estrela.

\section{AGRADECIMIENTOS}

Agradecemos la ayuda prestada por Henrique Garcia Pereira en la elaboración del manuscrito, así como a propósito de la metodología. A Rui Figueira por la colaboración para la obtención de los datos del Atlas do Ambiente. A todos las personas que contribuyeron a la formación de la base de datos, y especialmente a Ernestino Maravalhas. La investigación fue realizada con el apoyo de la Fundação para a Ciência e Tecnologia (Ministério da Ciência, Portugal) a través de la atribución de una beca de doctorado del programa PRAXIS XXI (BD9101/96).

\section{Referencias}

Austin, M. P., Pausas, J. G. \& Nicholls, A. O., 1996. Patterns of tree species richness in relation to environment in southeastern New South Wales, Australia. Australian Journal of Ecology, 21: 154-164.

Castro Praga, I., Moreno Sainz, J. C., Humphries, C. J. \& Williams, P. H., 1996. Strengthring the Natural and National Park system of Iberia to conserve vascular plants. Botanical Journal of the Linnean Society, 121: 189-206.
CVRM - Centro de Geo-Sistemas, 2000. Andad, ver. 6.0b. Instituto Superior Técnico, Av. Rovisco Pais, 1049-001 Lisboa, Portugal.

DENNIS, R. L. H., 1993. Butterflies and climate change. Manchester University Press. Manchester and New York. 301 pp.

DenNis, R. L. H., Shreeve, T. G. \& Williams, W. R., 1991. A multivariate approach to the determination of faunal structures among European butterfly species (Lepidoptera: Rhopalocera). Zoological Journal of the Linnean Society, 101: 1-49.

Dennis, R. L. H., Shreeve, T. G. \& Williams, W. R., 1995a. Taxonomic differentiation in species richness gradients among European butterflies (Papilionoidea, Hesperioidea): contribution of macroevolutionary dynamics. Ecography, 18: 27-40.

Dennis, R. L. H., Shreeve, T. G. \& Williams, W. R., 1995b. Affinity gradients among European butterflies: evidence for an historical component to species distributions. Entomologist's Gazette, 46: 141-153.

DENNIS, R. L. H. \& WiLliams, W. R., 1995. Implications of biogeographical structures for the conservation of European butterflies. In: A.S. Pullin (ed.). Ecology and Conservation of Butterflies. Chapman \& Hall. London: 211-229.

Dennis, R. L. H., Williams, W. R. \& Shreeve, T. G., 1998. Faunal structures among European butterflies: evolutionary implications of bias for geography, endemism and taxonomic affiliation. Ecography, 21: 181203.

D.G.A., 2000. Atlas do Ambiente. Direcção Geral do Ambiente, Portugal. http://www.dga.min-amb.pt/atlas, Abril 2000.

DE JONG, H., 1998. In search of historical biogeographic patterns in the western Mediterranean terretrial fauna. Biological Journal of the Linnean Society, 65: 99-164.

DE Jong, R., 1972. Systematic and geographis history of the genus Pyrgus in the Paleartic region. Tijdschrift voor Entomologie, 115(1): 1-121.

DE Jong, R., 1974. Notes on the genus Carcharodus (Lepidoptera, Hesperiidae). SHILAP Revista de Lepidopterología, 48(1): 1-9.

De Jong, R., 1978. Carcharodus tripolinus Verity, stat. Nov., une nouvelle espèce pour la faune d'Europe. Remarques au sujet de la notion d'espèce (Lepidoptera Hesperiidae). Linneana Bélgica, VII(4): 117-122.

ESRI - ENVIRONMENTAL Systems ReseARCh Institute, INC., 1998a. ArcInfo 7.2.1, CA, 380 New York Streets, Redlands, CA 92373-81000, USA.

ESRI- ENVIRONMENTAL SYSTEMS RESEARCH InSTITUTE, INC., 1998b. ArcView GIS Version 3.1, CA, $380 \mathrm{New}$ York Streets, Redlands, CA 92373-81000, USA.

García-Barros, E., Garcia-Pereira, P. \& Munguira, M. L., 2000. The geographic distribution and state of butterfly faunistic studies in Iberia (Lepidoptera: 
Papilionoidea, Hesperioidea). Belgian Journal of Entomology, 2: 111-124.

García-Barros, E., Gurrea, P., LuciáÑEz, M. J., Martín Cano, J., Munguira, M. L., Moreno, J. C., Sainz, H., SANZ, M. J. \& SimÓN, J. C., 2002. Parsimony analysis of endemicity and its application to animal and plant geographical distributions in the Ibero-Balearic region (western Mediterranean). Journal of Biogeography, 29: 109-124.

GarcíA-BArros, E. \& Munguira, M. L., 1999. Faunística de mariposas diurnas en España peninsular. Areas poco estudiadas: una evaluación en el umbral del Siglo XXI. SHILAP Revista de Lepidopterología, 27(106): 189202.

Garcia-Pereira, P., García-Barros, E. \& Munguira, M. L., 1999. Evaluación del conocimiento de la fauna de mariposas de Portugal Continental. SHILAP Revista de Lepidopterología, 27(106): 225-231.

GUTIERRÉZ, D., 1997. Importance of historical factors on species richness and composition of butterflies assembles (Lep.: Rhopalocera) in a northern Iberian mountain range. Journal of Biogeography, 24: 77-88.

GuTIERRÉZ, D., 1996. Variaciones espacio-temporales de los agregados de especies de lepidópteros ropalóceros en los Picos de Europa (Norte de España). Tesis doctoral. Departamento de Biología de Organismos y Sistemas, Universidad de Oviedo. 208 pp.

GutiérRez, D. \& MENÉNDEZ, R., 1995. Distribution and abundance of butterflies in a mountain area in the northern Iberian Peninsula. Ecography, 18: 209-216.

HewitT, G. M., 1999. Post-glacial re-colonization of European biota. Biological Journal of the Linnean Society, 68: 87-112.

HiLl, M. O., 1974. Correspondence Analysis: a neglected multivariate method. Applied Statistics, 23(3): 340-354.

KudRnA, O., 2002. The distribution atlas of European Butterflies. Oedippus, 20: 1-342.

LoBO, J., 1999. Individualismo y adaptación espacial: un nuevo enfoque para explicar la distribución geográfica de las especies. Boletín de la S.E.A., 26: 561-572.

Lobo, J. M., Praga, I. \& Moreno, J. C., 2001. Spatial and environmental determinants od vascular plant species richness distribution in the Iberian Peninsula and Balearic Islands. Biological Journal of the Linnean Society, 73: 233-253.

LuMARET, J-P. \& LOBO, J. M., 1996. Geographic distribution of endemic dung beetles (Coleoptera, Scarabaeoidea) in the Western Paleartic region. Biodiversity Letters, 3: 192-199.

Maravalhas, E., Garcia-Pereira, P. \& Schmitt, T., 2003. Monografia das borboletas de Portugal Continental (Continente). In: E. Maravalhas (ed.). As Borboletas de Portugal. Vento Norte. Vila Nova de Famalicão: 167324.
Martín, J. \& Gurrea, P., 1990. The peninsular effect in Iberian butterflies (Lepidoptera: Papilionoidea and Hesperioidea). Journal of Biogeography, 17: 85-96.

Martín, J. \& GuRreA, P., 1999. Areas de especiación en España y Portugal. Boletín de la Asociación Española de Entomología, 23(1-2): 83-103.

Mazel, R. \& LeEstmans, R., 1999. Seconde contribution à l'étude des relations entre Lepidea sinapis Linné et $L$. reali Reissinger en France, Belgique et autres contrées européennes (Lepidoptera: Pieridae). Linneana Belgica, XVII(4): 155-168.

Moreno Sainz, J. C., Castro Praga, I., Humphries, C. J. \& WiLliams, P. H., 1996. Strengthring the Natural and National Park system of Iberia to conserve pteridophytes. In: J. M. Camus, M. Gibby \& R. J. Johns (eds.). Pteridology in Perspective. Royal Botanic Gardens. Kew: 101-123.

Munguira, M. L., García-Barros, E. \& Martín, J., 1997. Plantas nutricias de los licénidos y satirinos españoles (Lepidoptera: Lycaenidae y Nymphalidae). Boletín de la Asociación Española de Entomología, 21(1-2): 29-53.

Myklestad, A. \& Birks, H. J. B., 1993. A numerical analysis of the distribution patterns of Salix L. species in Europe. Journal of Biogeography, 20: 1-32.

Olivares, J., Jutzeler, D. \& Leestmans, R., 1999. Arethusana boabdil (Rambur, 1840) de la prov. de Grenade, Andalousie: une bonne espèce! (Lep.: Nymphalidae, Satyrinae). Linneana Belgica, XVII(4): 135-154.

RiBeIro, O., 1986. Portugal - o Mediterrâneo e o Atlântico. Colecção "Nova Universidade", $N^{\circ} 13$. Livraria Sá da Costa. Lisboa. 188 pp.

StatSoft, InC., 1996. Statistica for Windows, Release 5.1 (computer program manual). StatSoft, Inc., Tulsa, OK, USA.

Thomas, J. A., 1989. The Conservation of butterflies in Temperate countries: past efforts and lessons for the future. In: R. I. Vane-Wright \& P. R. Ackery (eds.). The Biology of Butterflies. Princeton University Press. Princeton: 333-353.

Vives Moreno, A. 1994. Catálogo sistemático y sinonímico de los lepidópteros de la Península Ibérica y Baleares (Insecta: Lepidoptera). Segunda parte. Secretaría General Técnica. Ministerio de Agricultura, Pesca y Alimentación. Madrid. 775 pp. 
Apéndice 1.- Abreviatura de los nombres de las especies utilizadas para la presentación de los resultados.

Appendix 1.- Abbreviations of the species names used in the presentation of results.

\begin{tabular}{|c|c|c|c|c|c|}
\hline Especie & Cód. & Especie & Cód. & Especie & Cód. \\
\hline Adscita bolivari & bol & Fabriciana niobe & nio & Pandoriana pandora & pan \\
\hline Adscita hispanica & his & Gegenes nostrodamus & nos & Papilio machaon & mac \\
\hline Adscita jordani & jor & Glaucopsyche alexis & ale & Pararge aegeria & aeg \\
\hline Adscita notata & not & Glaucopsyche melanops & mel & Pieris brassicae & bra \\
\hline Adscita schmidti & sch & Gonepteryx cleopatra & cle & Pieris manni & man \\
\hline Aglais urticae & urt & Gonepteryx rhamni & Grh & Pieris napi & nap \\
\hline Aglaope infausta & $\inf$ & Hamearis lucina & luc & Pieris rapae & rap \\
\hline Anthocharis belia & Abe & Hesperia comma & com & Plebejus argus & $\arg$ \\
\hline Anthocharis cardamines & Aca & Hipparchia alcyone & Hal & Polygonia c-album & Pca \\
\hline Apatura ilia & Ail & Hipparchia fidia & fid & Polyommatus bellargus & bel \\
\hline Apatura iris & iri & Hipparchia semele & Hse & Polyommatus icarus & ica \\
\hline Aporia crataegi & cra & Hipparchia statilinus & sta & Polyommatus thersites & the \\
\hline Arethusana arethusa & are & Hyponephele lycaon & lyc & Pontia daplidice & dap \\
\hline Argynnis paphia & pap & Hyponephele lupina & lup & Pseudophilotes abencerragus & $a b c$ \\
\hline Aricia cramera & Acr & Inachis io & io & Pseudophilotes baton & Psb \\
\hline Aricia montensis & Amo & Iphiclides podalirius & pod & Pseudophilotes panoptes & Psp \\
\hline Azuritis reducta & red & Issoria lathonia & lat & Pyrgus alveus & alv \\
\hline Boloria dia & dia & Laeosopis roboris & rob & Pyrgus armoricanus & arm \\
\hline Boloria euphrosyne & eup & Lampides boeticus & boe & Pyrgus malvae & Pma \\
\hline Boloria selene & sel & Lasiommata maera & mae & Pyrgus onopordi & ono \\
\hline Brenthis daphne & Bda & Lasiommata megera & meg & Pyronia bathseba & $\mathrm{Pba}$ \\
\hline Brenthis ino & ino & Leptidea sinapis & $\sin$ & Pyronia cecilia & $\mathrm{cec}$ \\
\hline Brintesia circe & cir & Leptotes pirithous & pir & Pyronia tithonus & Pti \\
\hline Cacyreus marshalli & mar & Libythea celtis & cel & Satyrium esculi & esc \\
\hline Callophrys avis & avi & Limenitis camilla & cam & Satyriumilicis & ili \\
\hline Callophrys rubi & rub & Lycaena alciphron & alc & Satyrium spini & spi \\
\hline Carcharodus alceae & Cal & Lycaena hippothoe & hip & Satyrus actaea & Sac \\
\hline Carcharodus baeticus & bae & Lycaena phlaeas & phl & Spalia sertorius & ser \\
\hline Celastrina argiolus & Car & Lycaena tityrus & tit & Syrichtus proto & pro \\
\hline Charaxes jasius & jas & Lycaena virgaureae & vir & Thecla quercus & que \\
\hline Coenonympha arcania & arc & Maculinea alcon & Mal & Thymelicus acteon & Tac \\
\hline Coenonympha dorus & dor & Maniola jurtina & jur & Thymelicus lineola & lin \\
\hline Coenonympha glycerion & gly & Melanargia ines & ine & Thymelicussylvestris & syl \\
\hline Coenonympha pamphilus & pam & Melanargia lachesis & lac & Tomares ballus & bal \\
\hline Colias alfacariensis & alf & Melanargia occitanica & Moc & Vanessa atalanta & ata \\
\hline Colias crocea & cro & Melanargia russiae & rus & Vanessa cardui & Vca \\
\hline Cupido Iorquinii & lor & Melitaea aetherie & aet & Vanessa virginiensis & Vvi \\
\hline Cupido minimus & $\min$ & Melitaea athalia & ath & Zerynthia rumina & rum \\
\hline Cyaniris semiargus & Cse & Melitaea cinxia & $\operatorname{cin}$ & Zizeeria knysna & kny \\
\hline Danaus plexippus & ple & Melitaea deione & dei & Zygaena fausta & fau \\
\hline Erebia triaria & Etr & Melitaea didyma & did & Zygaena hilaris & hil \\
\hline Erynnis tages & $\operatorname{tge}$ & Melitaea parthenoides & par & Zygaena lavandulae & lav \\
\hline Euchloe belemia & Ebe & Melitaea phoebe & pho & Zygaena nevadensis & nev \\
\hline Euchloe crameri & Ecr & Melitaea trivia & tri & Zygaena occitanica & Zoc \\
\hline Euchloe tagis & $\operatorname{tgi}$ & Mesoacidalia aglaja & agl & Zygaena rhadamanthus & Zrh \\
\hline Euphydryas aurinia & aur & Nymphalis antiopa & ant & Zygaena sarpedon & sar \\
\hline Euphydryas desfontainii & des & Nymphalis polychloros & pol & Zygaena trifolii & Ztr \\
\hline Fabriciana adippe & adi & Ochlodes venata & ven & & \\
\hline
\end{tabular}

